
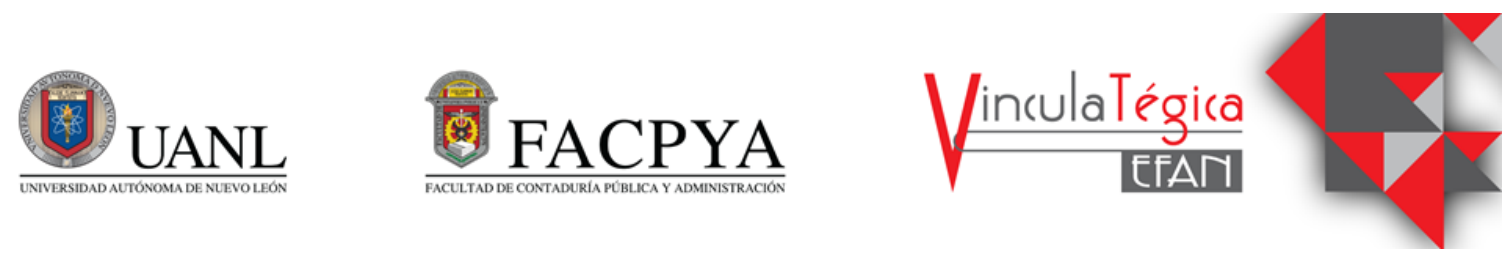

\title{
Estrategias de ventas en redes sociales en el aseguramiento de calidad y calidez
}

\author{
Vibiana Curiel Guzmán ${ }^{1}$ \\ ${ }^{1}$ Universidad de Guadalajara, vibianacuriel@gmail.com, Av.Universidad 203, Delegación Ixtapa, Jal. \\ 48280, Puerto Vallarta, Jal.
}

\author{
Información del artículo revisado por pares \\ Fecha de aceptación: junio-2021 \\ Fecha de publicación en línea: diciembre-2021 \\ DOI: https://doi.org/10.29105/vtga7.1-91
}

\begin{abstract}
Resumen
Es importante conocer que teorías existen, que tanto falta por escribir y conocer, enfocándome en procesos de trabajo para la mejora continua, gestionando servicios a través de la mercadotecnia y la competitividad en Puerto Vallarta, hoy me gustaría escribir sobre:¿Qué son las emociones? Hablemos de marketing emocional, como compra el cliente en la actualidad, las emociones son el motor que mueve la sociedad, las que humanizan al ser humano. Hablar de Innovación en valor es hablar sobre la piedra angular de la estrategia del océano Azul la innovación en valor se crea en la región en la cual los actos de una compañía inciden favorablemente sobre su estructura y sobre la propuesta de valor para los compradores. Las economías se logran al eliminar y reducir las variables sobre las cuales compite una industria. El valor para los compradores se aumenta al buscar y crear elementos que la industria nunca ha ofrecido. Con el tiempo, los costos se reducen todavía más cuando entran a operar las economías de escala debido al mayor nivel de ventas emanado del valor superior. (Kim, 2008)
\end{abstract}

Palabras clave: hábitos de compra, lealtad de los clientes, creatividad, innovación a través del servicio, modelos de negocios, pensamientos disruptivos, marketing emocional, competividad internacional.

Códigos JEL:

M, Administración de empresas, y marketing, M31 Marketing, A31 Trabajos de varias materias.

\begin{abstract}
It is important to know what theory exists, how much has been written, and how much remains to be written and learned, focusing on work processes for continuous improvement, managing services through marketing and competitiveness in Puerto Vallarta, and today I would like to write about : What are emotions?, in order to speak about emotional marketing it is important to find out, in the human being this emotions are the engine that moves society.

To talk about Innovation in value is to talk about the cornerstone of the strategy of the Blue Ocean. Innovation in value is created in the region in which the actions of a company have a favorable impact on its structure and on the value proposition for buyers. Economies are achieved by eliminating and reducing the variables on which an industry competes. Value for buyers is increased by searching for and creating items that the industry has never offered. Over time, costs are further reduced when economies of scale come into play due to the higher level of sales emanating from superior value.
\end{abstract}

Palabras clave: hábitos de compra, lealtad de los clientes, creatividad, innovación a través del servicio, modelos de negocios, pensamientos disruptivos, marketing emocional, competividad internacional.

Códigos JEL:

M, Administración de empresas, y marketing, M31 Marketing, A31 Trabajos de varias materias 


\section{INTRODUCCIÓN}

"Hablar del marketing emocional en servicios turísticos y hotelería como una herramienta estratégica basada en la experiencia del consumidor, me ha llevado a analizar la teoría del libro del océano azul, del Sr. Kim Chan. Algunas empresas aseguran que existen 3 pilares en el marketing emocional"( (Fillol, 2020).

\section{Vínculo afectivo marca- público}

"No solo el consumidor o cliente vive experiencias, también las marcas lo hacen a través de estos que, afortunadamente para las marcas inteligentes, sabrán sacarle provecho de esa retroalimentación que el consumidor es capaz de dar". (Fillol, 2020)

"Por una parte, la marca genera un producto que cubre las necesidades de sus consumidores, y por otra, el consumidor y/o cliente está satisfecho, no solo por el producto porque cubre sus necesidades, sino por las emociones y sensaciones que este le produce. Filosofía de vida de la empresa, valores en la empresa." (Fillol, 2020)

\section{El marketing, sensaciones y emociones}

Tenemos que posicionar nuestra marca a través de la mercadotecnia, ejemplo, la curiosidad. El proceso creativo que te jala $\mathrm{y}$ te da influencia para ver tu historia, o ver tu página web. (Fillol, 2020).

\section{Experiencias}

"Atraves de las experiencias vivimos sensaciones y sentimos emociones. y eso nos lleva a tomar decisiones."

Las empresas en la actualidad buscan crear experiencias memorables en cada visita a un hotel, en cada compra, en cada servicio, porque las experiencias se quedan guardadas en el cliente, y el preferirá tu marca mientras no haya otra empresa que te gane ese lugar.

Cada publicidad debe estar impregnada de nuestros valores, ejemplo el valor de la diversión. como agregas valor a tus comunicaciones a tus redes.

Principios que definen la estrategia del océano azul y las diferencias de la estrategia basada en la competencia (Kim, 2008).Construir confianza y compromiso. Se busca que gane el cliente con el servicio, los empleados trabajen felices, y los accionistas tengan una buena utilidad. El enfoque dejar huella y encontrar la manera de repetirlas sistemáticamente.

Al eliminar elementos costosos reduce precio, logrando ir creando una diferenciación y es una muy buena estrategia mantener un buen precio. Minimizar el riesgo en lugar de propiciarlo.

La competencia pierde importancia al explorar más allá de las 6 fronteras convencionales de la compañía con el fin de abrir océanos azules comercialmente importantes. Es esta búsqueda constante de la diferenciación y el bajo costo.

Figura 1-2 dinámica de diferenciación y bajo costo sobre la cual se apoya la innovación en valor. 


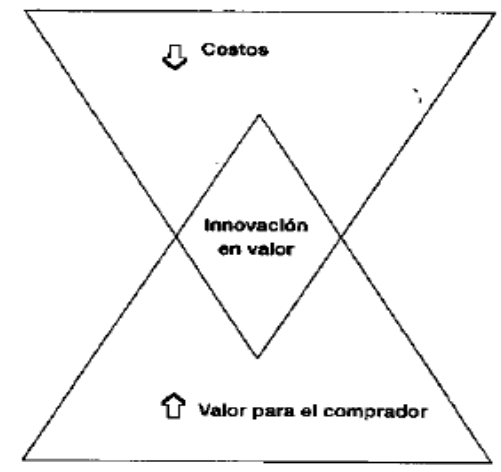

Fuente: (Kim, 2008)

\section{FIGURA 1-3}

\section{La estrategía del océano rojo frente a la estrategia del oceano azul}

\begin{tabular}{|c|c|}
\hline Eetratepla del ocedano ralo & Estratogilu dal ocibano azul \\
\hline $\begin{array}{l}\text { Competis on el ospacio existente del } \\
\text { mercado. }\end{array}$ & $\begin{array}{l}\text { Crear un espacio sin competencia en al } \\
\text { mercado. }\end{array}$ \\
\hline Vencer a la competencle. & $\begin{array}{l}\text { Hacer que la competencia pierda toda } \\
\text { importancia. }\end{array}$ \\
\hline $\begin{array}{l}\text { Explotar la demanda exlstenta en el } \\
\text { mercado. }\end{array}$ & Grear y capturar nueva dertarnda. \\
\hline $\begin{array}{l}\text { Elegir entre le disyuntha de vator o } \\
\text { costo. }\end{array}$ & Romper la disyuntiva de valor a costo. \\
\hline $\begin{array}{l}\text { Allnear todo el siatema de las activi- } \\
\text { dedes de una empresa con la dectsión } \\
\text { astretejica de la diferenciaclón o del } \\
\text { bajo costo. }\end{array}$ & $\begin{array}{l}\text { Alinear todo el sistema de las activida- } \\
\text { des de una empresa con el propósita de } \\
\text { lograr diferenciación y bajo costo. }\end{array}$ \\
\hline
\end{tabular}

(Kim, 2008)

Problema a investigar:

Los hechos indican que el aumento de estrategias de ventas en las redes sociales impacta en el aumento de las quejas de clientes, se refleja en el volumen de ventas, en las ganancias para intermediarios, lo anterior lleva a la baja competitividad de la empresa, además no se ha logrado la optimización de ventas, a través de redes sociales.

El volumen tan alto de trabajo y los procesos de ventas en las redes sociales que se llevan a cabo en los hoteles no permiten el adecuado

\section{MARCO TEORICO}

servicio a clientes, por lo cual existe un volumen de quejas por resolver en cada grupo, y se pierden los clientes.

Problemática:

$\checkmark$ Demasiada oferta hotelera, caída de la demanda, costos operacionales, transiciones sociales, condiciones económicas, redes sociales y su impacto en las ventas,
De acuerdo a (Deming, 1950), los procesos de calidad de la mejora continua tienen que ser 
establecidos en las empresas para lograr la optimización de los servicios al cliente.

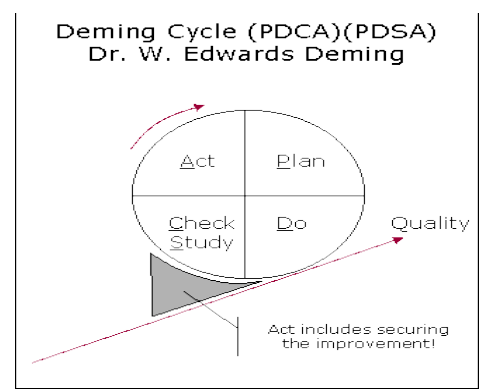

Fuente: (Remondegui, 2014)
En la hotelería es importante establecer indicadores de calidad y llevar una medición de la satisfacción del cliente.

Teoria de las redes sociales (Ochoa, 2021)

Disrupción a la velocidad de las redes

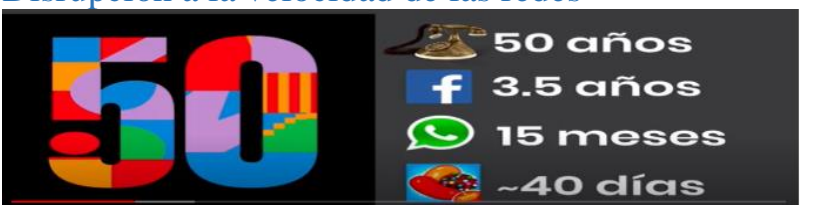

Fuente: Video de Microsoft El camino de la Disrupción

Hanói Morillo, Líder Regional IBM Digital Transformación \& Innovación.

Las empresas están desapareciendo, o cambiamos o nos quedaremos obsoletos.

Customer center, tendencia enfocada al cliente

\section{Redes sociales: herramienta de marketing internacional en el sector hotelero}

Marketing internacional

"De acuerdo con datos de la Internet Society (ISOC), en 2015 se tienen aproximadamente a tres mil millones de personas conectadas a Internet en todo el mundo, una cifra que se traduce en que $42 \%$ del orbe ya cuenta con algún tipo de acceso frecuente a la autopista de la información y la comunicación (ISOC, 2015). Así mismo, se puede decir que las redes sociales se consolidan como plataforma de promoción internacional empresarial con un gran potencial, en el que no únicamente las grandes compañías obtienen provecho de esta tecnología, pues cualquier organización
Qué tipo de cosas está pidiendo el cliente, un sistema que te permita responder a ellos rápidamente.

puede, sin importar su tamaño, conectar con consumidores, clientes y prescriptores de todo el mundo, abrir nuevos mercados, reforzar su imagen internacional de marca, reforzar el vínculo con su público objetivo en cada país con mensajes personalizados y respuestas en tiempo real."

Para Lázaro (2014), "la estrategia de comunicación y marketing en redes sociales debe estar integrada en la propia gestión internacional del negocio, ya sea desde el punto de vista de atención al cliente, posicionamiento de marca o consolidación de mercados. Así como también tomar en consideración el componente intercultural, lo cual es fundamental en toda gestión 
empresarial multinacional, que permita conocer y comprender las diferencias culturales del interlocutor y actuar en consecuencia, ya sea presencialmente, online o a través de una red social. Por tanto, la red social constituye un elemento más de la estrategia de marketing y promoción internacional. No sustituyen ninguna otra herramienta, simplemente la complementan. También, es necesario tomar en cuenta la posible existencia de legislaciones nacionales en cuanto a publicidad, promoción y venta de determinados productos".

(rperiplo.uaemex.mx)

\subsection{Justificación}

En la hotelería es importante establecer indicadores de calidad y llevar una medición de la satisfacción del cliente.

Es importante entonces que el servicio al cliente también se desprenda que el hotel pueda atraer y depende en mayor medida gradualmente de sus esfuerzos mercadológicos, esto es, capturar la atención de los clientes en forma cada más directa. De esta manera, la institución tendrá más eficiencia en su devenir económico y aplicara estos esfuerzos en ofrecer servicios de mejor calidad a sus huéspedes para redundar en clientes repetitivos.

\section{Bajar los costos operativos y las comisiones}

$\mathrm{Al}$ incursionar directamente el hotel en el mercado competirá obviamente con el factor precio. Dicho factor, es estratégico dado que la empresa puede de esta manera tomar segmentos de mercado directamente, sin intermediarios, evitando así pagar las comisiones onerosas que disminuyen su utilidad y demeritan la calidad de sus servicios. Por otra parte, los costos operativos impactaran en menor medida las condiciones financieras y permitirán mayor solvencia económica.

\subsection{Pregunta de investigación}

¿Cuáles son las limitantes que interfieren en las ventas y en la calidad de los servicios al cliente a través de las redes sociales para incrementar las utilidades de los hoteles de 5 estrellas de Puerto Vallarta?

\subsection{Objetivo General}

Describir el Modelo de ventas directas a través de redes sociales que minimizan las quejas y brinda mayor atención al cliente, lo cual lleva a incrementar la satisfacción del turista que hace uso de este medio, y también, la rentabilidad de los hoteles de 5 estrellas de Puerto Vallarta.

Bajo qué tipo de esquema de trabajo se permite un aseguramiento de la calidad.

\subsubsection{Objetivos específicos}

1. Establecer el segmento de mercado meta para los hoteles de 5 estrellas de Puerto Vallarta, tanto nacional como internacional.

2. Registrar la oferta o planta turística de categoría 5 estrellas de Puerto Vallarta.

3. Describir el posicionamiento actual en redes sociales de la oferta o planta turística local de categoría 5 estrellas.

4. Describir cómo se puede obtener beneficios por el uso de las redes sociales y a la vez tener un buen servicio al cliente con calidad y calidez.

¿Cómo cambiara nuestro espacio y percepción del mundo, ahora que se tendrán que cambiar hábitos, guardar la sana distancia, en cuanto tiempo se estima se recupere este destino turístico?

Si hay dinamismo de recursos de las empresas hoteleras para dar respuesta a los mercados y se gestara un nuevo modelo de negocio.

Encontrar si los hoteles pueden mejorar su servicio al cliente y obtener beneficios usando las plataformas sociales, en una forma eficiente, creando estrategias de 
atención personalizada, calidad y calidez, manteniendo un liderazgo en redes sociales constante, creando contenido y contestando las preguntas de los usuarios poniendo atención y respondiendo a los comentarios

\section{METODOLOGÍA}

¿Qué ruta piensa seguir en la investigación?

1. Diseño de la Investigación Aplicada, cualitativa, descriptiva

2. Selección de la muestra Encontrar el cliente que compre cada marca 5 estrellas

3. Recolección de datos A través de encuestas

4. Análisis de datos graficar en forma anual, que porcentaje es repetitivo, y como seguir manteniéndolo para obtener un equilibrio en las ventas.

\section{Antecedentes:}

La ciudad de Puerto Vallarta, principalmente la hotelería, agencias de viajes, transportistas, restauranteros y comerciantes en general, se han visto fuertemente impactada en su economía ante esta pandemia del COVID. Ahora todo es cambio, se establecen protocolos de seguridad y nuevas programas corporativos en las diversas cadenas hoteleras para volver al trabajo en una realidad nueva, aprendiendo a vivir y trabajar con el sector turístico con esta pandemia.

Puerto Vallarta y la hotelería en general se ha puesto a prueba ante esta situación económica, su situación ante el turismo, y enfrenta una crisis económica de las más fuertes que ha tenido.

Ahora más que nunca es necesario INNOVAR, cambiar la forma como se trabaja, salir a la calle, renovados, y lograr hacer el cambio que se necesita. La competitividad internacional sigue estando ahí, pero hay muchas ventajas que se tienen que capitalizar, buscando la sustentabilidad.

Social media

El desarrollo de una buena estrategia de social media, es una de los factores más importantes a la hora de mantener una buena comunicación con el cliente. Las redes sociales han venido a revolucionar los mercados, y nos ofrecen un plus añadido en la gestión de reputación online, y siempre se puede aprovechar ese potencial.

La social media se ha convertido en una parte muy importante, en la comercialización de viajes, las relaciones públicas y el cuidado de la reputación online. Un gran porcentaje de los usuarios examinan canales sociales como Facebook, Instagram, Linkedin, Twitter o Google Plus buscando encontrar opiniones acerca de los alojamientos en los que van a hospedarse.

La atención y satisfacción del cliente son puntos que no se deben de olvidar en ningún negocio.

Como se diseñan experiencias centradas en el cliente

El banco ID BANK, cambio sus horarios fue el primer banco que se adapta a cuando el cliente puede asistir, incluso pueden entrar con su mascota.

No tenemos que ser Amazon para brindar servicios al cliente. No se vale que le vendamos al cliente lo que nosotros queremos vender sino vender lo que el cliente quiere.

Como generamos a los nuevos líderes, una nueva generación de liderazgo, en una era digital, acelerar la cultura de la innovación, como generar modelos dinámicos de gestión de personas, como reinventamos al talento.

\section{Creatividad enfocada al negocio}

La creatividad, pensamiento original, imaginación constructiva, pensamiento divergente o pensamiento creativo, es la generación de nuevas ideas o conceptos, o de nuevas asociaciones entre ideas y conceptos conocidos, que habitualmente producen soluciones originales. (Silva, 2021)

La utilización de las redes sociales enfocadas a ventas, contratando en los hoteles especialistas en el manejo de las redes, e managers, social media manager...etc. 


\section{El diseño de actividades}

Segmentar,
Valores, el compromiso social, conectarnos con los problemas de la sociedad, conectar con los intereses de las personas, que cosas saben, si son artistas, apoyarlos para que creen sus espacios de arte. El ser humano como cliente, hacerlo que se sienta y este seguro.

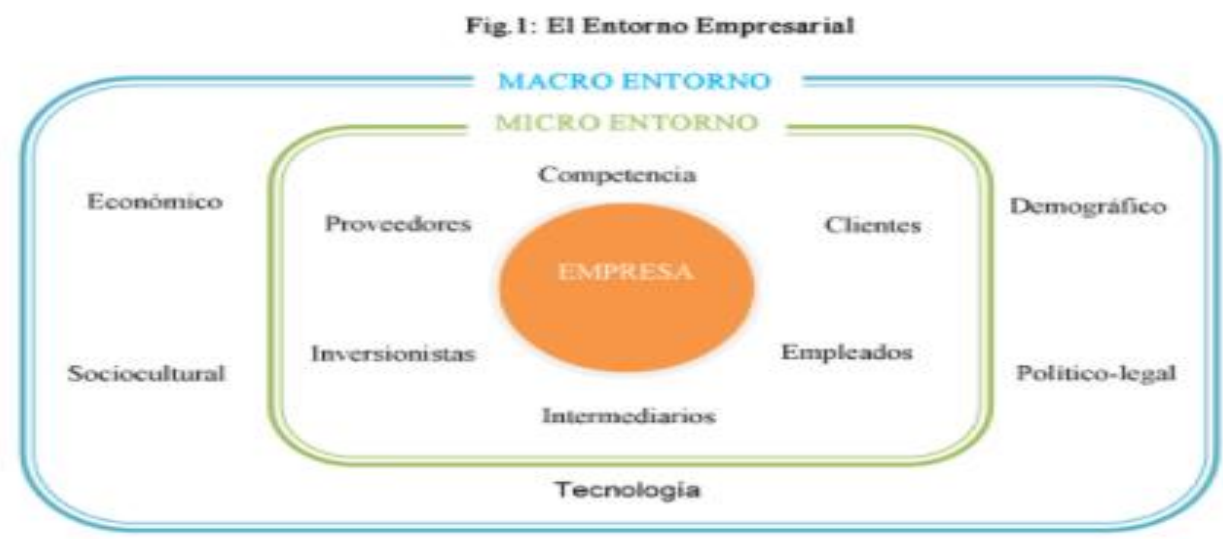

Fuente: elaboración propia en función a la teoria de la administración moderna

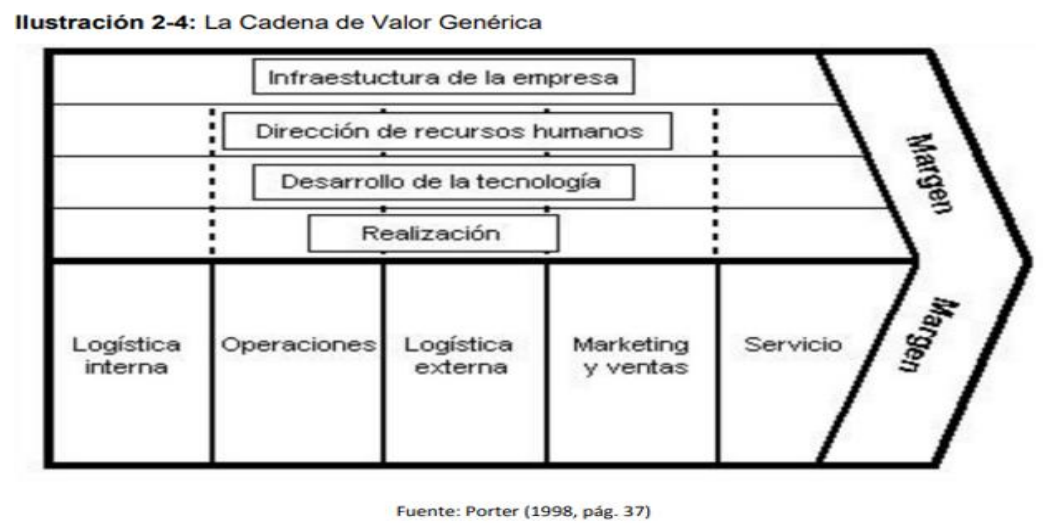

\section{FACTORES SOCIALES:}

Las redes sociales, tienen sus ventajas para las empresas, ya que el cliente puede compartir lo que piensa, y desea comentar para que otros lectores lo vean. Puede compartir su experiencia placentera obtenida en su estancia, lo que impacta directamente en otras personas del mismo país y del extranjero que lean el mensaje publicado por uno de los huéspedes que ha quedado satisfecho con el servicio obtenido. Es importante cuidar y dar respuesta analizando los puntos que se mencionan en negativo, ya que de no estar atento pueden repercutir en la imagen de la empresa, teniendo en cuenta que se trata de una red mundial y ésta puede llegar a recorrer en instantes a miles de personas conectados a estos medios digitales. Se sugiere mantener las redes sociales activas y con participación constante, revisando los comentarios de los huéspedes y sus respuestas si han evaluado como regular los servicios habra que revisar los porqués. Es importante preguntar a la audiencia como arreglarlo. Debe procurarse preguntar a la audiencia lo que les interesa o necesitan que sea tomado en cuenta por las organizaciones.

Estas plataformas pueden ser el vínculo ideal para fomentar la interacción e incentivar el 
interés de participación que puede representar encontrar algunas áreas de mejora, buscando la creación de valor en el servicio ofertado. Las redes sociales son un canal estratégico para la interacción con los clientes y la comunicación $\mathrm{y}$ oferta de los servicios de las empresas hoteleras, por lo tanto las redes sociales pueden convertirse en un canal estratégico para la comunicación de los servicios de las empresas hoteleras, por lo tanto es necesaria la creación de un programa o plan que respalde el seguimiento de las iniciativas necesarias para el alcance de las metas.

Es importante que las empresas se dediquen a conocer mejor a su audiencia en las redes sociales y que este medio no solamente copie lo que hace la competencia, será importante trazar objetivos identificables con la orientación al cliente, que les permita trazar un camino hacia la fidelización de sus consumidores.

La empresa ganadora será la que este centrada en el cliente, que ha digitalizado y automatizado todos los procesos manuales, entiende como maximizar sus resultados a través de tecnologías y estrategias de innovación y lleva a sus equipos una cultura ágil capaz de adaptarse a los cambios antes de que sucedan.

La satisfacción de clientes en el hotel Marriot, 5 estrellas como referencia, de acuerdo a Trip Advisor es de 4.5. El éxito de una estrategia de fidelización depende de que el proceso para alcanzar la fidelidad del huésped esté bien definido en sus objetivos y de que realmente se lleve a la práctica en la gestión diaria del hotel.

Mercado, avances tecnológicos en hoteles, nuevas tendencias en la tecnología para hoteles

presencia en los medios sociales,el diseño de ofertas personalizadas.

Maximizar la utilización de diferentes canales de venta directa, Extender servicios a través de aplicaciones móviles.,comercio a través de Facebook, Las video-conferencias. El servicio WiFi, disponible en mas lugares, más servicios adaptados a las nuevas tecnologías.

\section{MODELOS DE NEGOCIOS}

FIGURA 1.1 Modelo simple del proceso de marketing

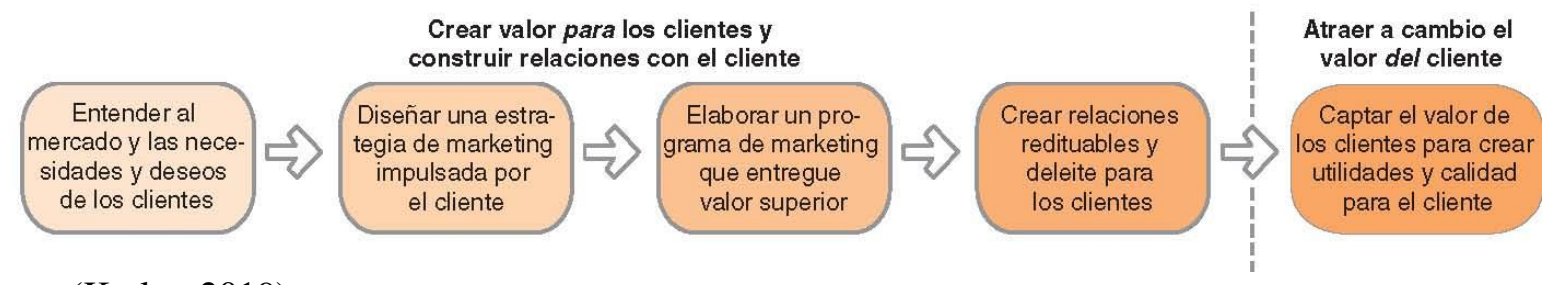

Fuente: (Kotler, 2019)

Los aspectos intangibles, derivados del pensamiento creativo, son elementos fundamentales de la oferta de los productos y servicios (Casani, 2018)

1.2. Creatividad en los modelos de negocio

Un modelo de negocio describe la lógica sobre cómo una organización crea, entrega y captura valor. Los modelos de negocios son básicamente historias que explican cómo trabajan las organizaciones, indicando quiénes son nuestros clientes, cómo generamos utilidades, cuál es la lógica económica subyacente que nos permite entregar valor a los clientes a los que nos dirigimos a un costo apropiado. Es una descripción sistémica de cómo es que las piezas de un negocio mejoran. (Jair, 2020) 


\section{Creatividad en los modelos de negocio}

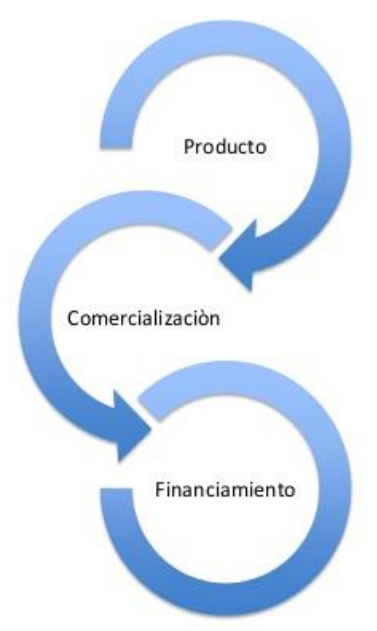

Figura 1.3. Modelos de negocio

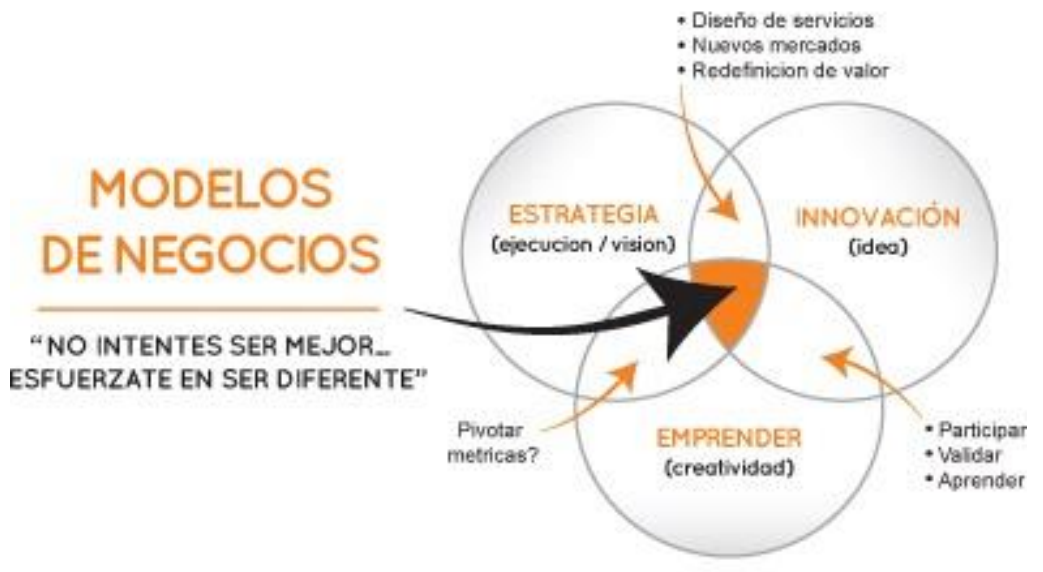

Fuente(Antonio, 2020)

ANALISIS FODA DE LA SITUACIÓN

Fortalezas

Posicionamiento como destino turístico, calidad en el servicio a clientes, negocio repetitivo

\section{Oportunidades}

Crecimiento en posicionamiento en redes sociales, mayor publicidad en redes sociales, y a nivel internacional, marketing emocional para generar fidelidad en el cliente, contar con practicantes que lleven la publicidad y les fortalezca

\section{Debilidades}

Diversidad en sus formas de administrar cadenas hoteleras y hoteles privados, No contar con personal capacitado en manejo de redes y publicidad en tiempo y forma, costos involucrados en las ventas 


\section{Método}

\section{Amenazas}

La apertura de más hoteles, la contaminación ambiental, los fenómenos atmosféricos impredecibles, altos costos en mantener nómina base y baja ocupación
ENCUESTA a través de cuestionario para conocer las preferencias del cliente.

Se hizo encuesta a estudiantes como prueba para analizar qué opinan sobre 10 preguntas. 
Resultados de encuesta aplicada: PREGUNTAS QUE LE HARIA AL CLIENTE

1. Datos demográficos

Edad ___ (Generación)

Ocupación Nacionalidad

Género, femenino, masculino.

2. Que lo llevo a buscar el destino Puerto Vallarta?

\begin{tabular}{lll}
$\circ$ & Playa & Cultura \\
$\circ$ & Conectividad & Negocios \\
$\circ$ & Otro_ & \\
\hline
\end{tabular}

3. Hubo alguna marca en la hotelería con la que usted se sintió atraído?
○ Sheraton
Westin
- Marriot
o W.
St. Regis
OTRO

4. Cuáles son esas características que ofrece esa marca por las que usted elige hospedarse ahí. (Diferenciadores que nos ayuden a encontrar porque compra el cliente)

○ Lujo para gente joven

- Reencuéntrate, reconéctate

○ Familiar
Bienestar

Lujo tradicional

5. Que te atrajo a tu elección

- Aventura y nuevas experiencias Negocio

- Convivencia familiar Contacto con la naturaleza

- Lealtad

6. Que es lo que refresca tus sentidos?

- Visita a lugares ecológicos Arte en Puerto Vallarta

○ Cultura Huichol Diversidad de actividades

7. ¿Qué valores agregados prefieres encontrar en el servicio?

- Sensaciones que te conectan

- Gastronomía

8. Como se enteró de Puerto Vallarta

- Radio

- Medios digitales

9. Como es tu viaje

○ Familia

Pareja

- Sencillo
Disfrutar del Spa

Que se permita vacacionar con tu mascota

10. Como elijes la marca del hotel para hospedarte 
- Valor por el precio pagado

- Tendencias

- Última tecnología
Innovación

Vanguardia,

\section{Respuestas:}

\section{DATOS DEMOGRAFICOS1. Datos personales.}

Edad

13 respuestas
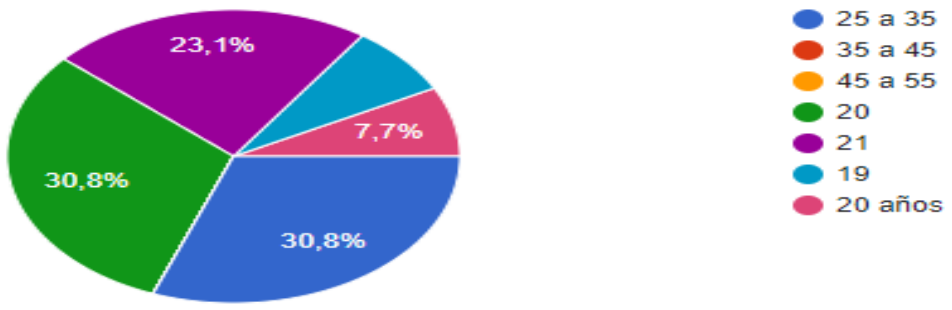

1A

Genero

13 respuestas

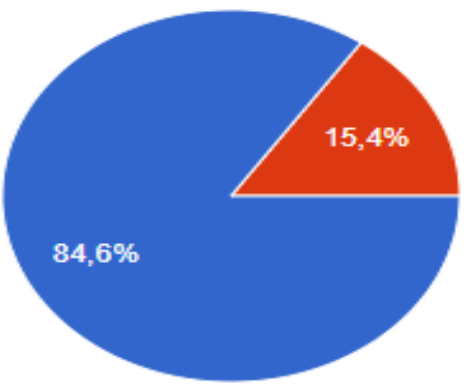

Prefiero no decirlo

2. 
¿Qué te motivó a visitar el destino Puerto Vallarta?

13 respuestas

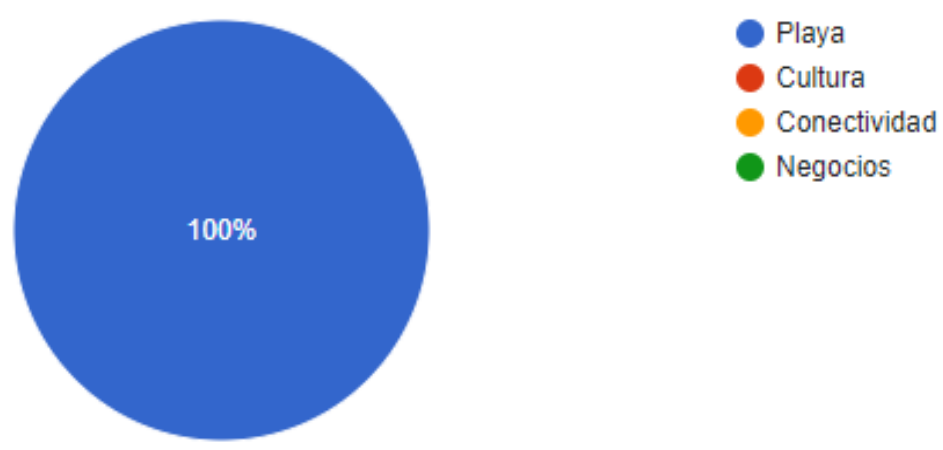

3.

¿Hubo alguna marca en la hoteleria con la que usted se sintió atraido? 13 respuestas
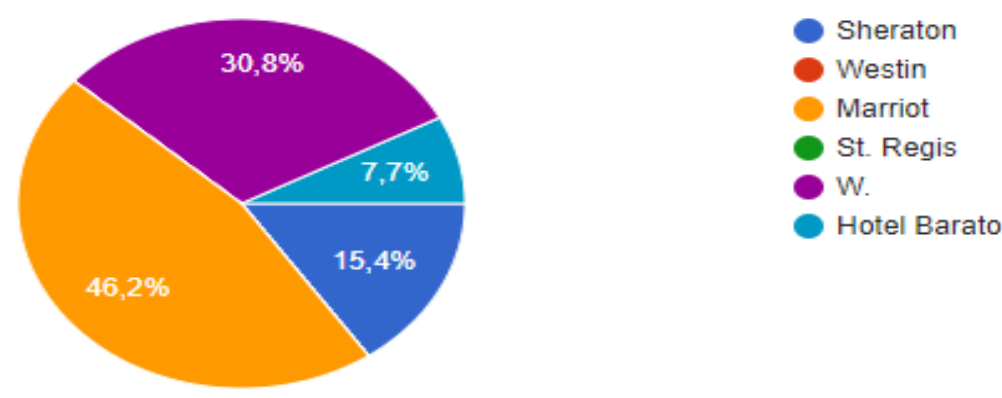

4.

¿Cuáles son esas caracteristicas que ofrece esa marca por la que usted eligió hospedarse ahi?

13 respuestas

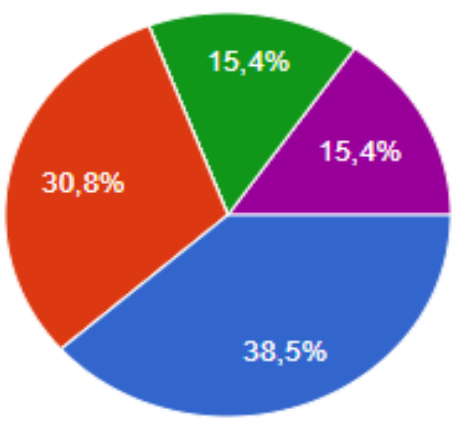

Lujo para gente joven

Bienestar

Reencuentrate / Reconéctate

Lujo tradicional

Familiar 
5.

¿Qué te trajo a tu elección?

13 respuestas

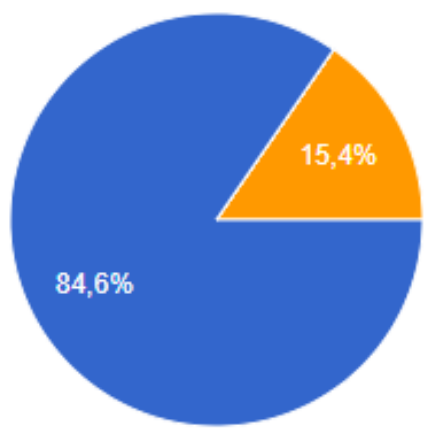

Aventura y nuevas experiencias

Negocio

Convivencia familiar

Contacto con la naturaleza

Lealtad

6.

¿Cómo se enteró de Puerto Vallarta?

13 respuestas

7.

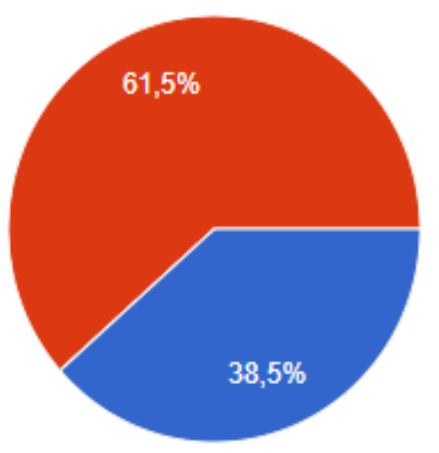

Recomendación

Redes sociales

Televisión

Radio

¿Qué es lo que refresca tus sentidos?

13 respuestas

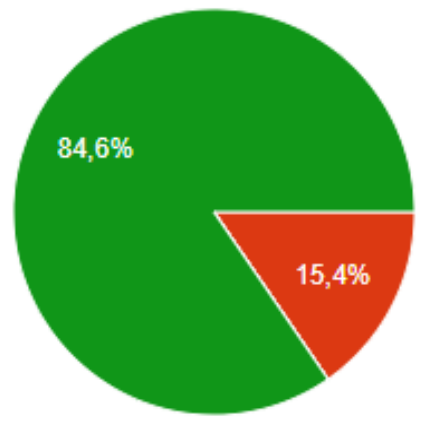

Visita a lugares ecológicos

Arte en Puerto Vallarta

Cultura Huichol

Diversidad de actividades 
8.

¿Qué valores agregados prefieres encontrar en el servicio?

13 respuestas

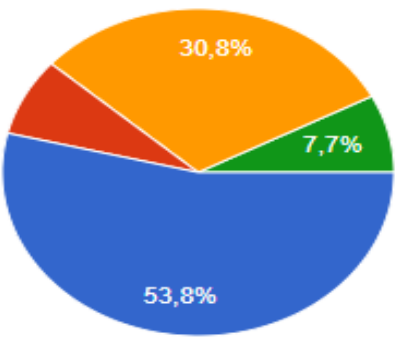

9.

¿Cómo fue tu viaje?

13 respuestas

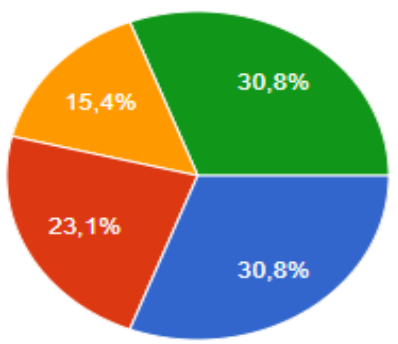

Sensaciones que te conectan

- Disfrutar del Spa

Gastronomía

Vacacionar con tu mascota en el hotel

10.

¿Qué diferenciadores en la marca del hotel buscas para hospedarte?

13 respuestas

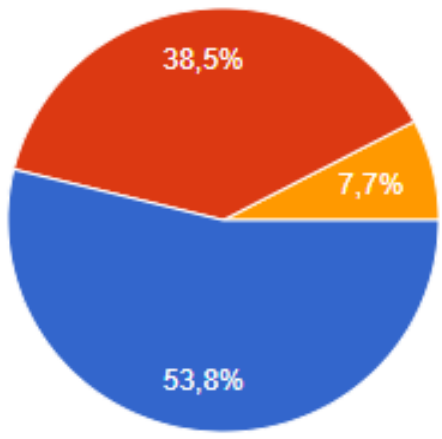

- Grupal 


\section{CONCLUSIONES:}

Puerto Vallarta se puede convertir en una gran ciudad artística, vienen muchos músicos, cantantes de ópera, artistas de cine, pintores, gente que ahora tiene una escuela tipo museo de arte, todo esto genera más turismo para la comunidad.

$\checkmark$ Desarrollo capital humano enfocado en los procesos de calidad en el servicio a clientes

Capacitación constante de toda la gente en el destino turístico en el área de servicio al cliente, y estableciendo sistemas de calidad en los servicios turísticos.

\section{Acciones a considerar en el trato a}

\section{clientes}

* Aumentar la participación en el mercado

* Posicionar la marca

* Mejorar el posicionamiento del destino turístico

* Generar más ambientes de neuroeducacion, conectando las personas con las sensaciones, asociadas al tema, conectar con las emociones, que la gente conozca las perspectivas de servicio, en todas las áreas que tenga el contacto el cliente.

* Mejorar las condiciones sociales del trabajador

Lo que nos va permitir hacer todos estos cambios es la tecnología.

1. celular, que tengamos respuesta a correo, entrar a páginas web

2. Nube, lograr tener respaldos, y datos de nuestras transacciones, base de datos, y poder acceder en tiempo real.

3. Inteligencia artificial, como se puede cuadrar las plataformas

4. Si vemos que el mundo se está descentralizando y cada vez hay más trabajos, quedarse con un modelo en tiempo real, plataforma blue chain, más eficientes a otra escala, manejando las finanzas y optimizando recurso tiempo.

\section{Mantener estándares e ir cuidando cada detalle:}

$\checkmark \quad$ La amabilidad y el trato Cortes son importantes en la primer impresión

$\checkmark \quad$ En el caso de grupos y convenciones o bodas, hacer reuniones con los clientes para explicarles las ventajas de llegar a Puerto Vallarta y todas las excursiones.

$\checkmark$ Capacitar al personal para que hable inglés para que atienda correctamente las llamadas que se reciban en la oficina

$\checkmark \quad$ Las estrategias de comercialización y porque son tan importantes:

Estrategia significa elegir de forma precisa la forma de competir Jack Welch (Arthur A. Thompson Jr, 2007)

La esencia del servicio, nos ha hecho regresar a lo básico, a que nos sintamos bien. Lo básico te lleva al éxito, la regla de oro en el servicio, trata a los demás como tú deseas ser tratado.

Las redes sociales pueden convertirse en un canal estratégico para la comunicación de los servicios de las empresas hoteleras, por lo tanto es necesaria la creación de un programa o plan que respalde el seguimiento de las iniciativas necesarias para el alcance de las metas. En la actualidad, es necesario que las organizaciones busquen conocer mejor a su audiencia en las redes sociales y que este medio no solamente aparezca como una división construida a partir de copiar lo que hace la competencia,

debe realizarse como una necesidad de desarrollar objetivos identificados con la orientación al cliente, que les permita trazar un camino hacia la fidelización de sus consumidores. 


\section{Bibliografía:}

http://www.fundacionorange.es/wpcontent/uploads/2016/05/eE_La_transformacion_digital_del_sector_turistico.pdf https://www.competitividadyestrategia.com/12-pilares-de-la-competitividad.html https://aecit.org/uploads/public/DOCUMENTO.covid-19\%20y\%20turismo.pdf https://www.leshoteliers.com/redes-sociales-estrategia-marketing-hotelero/ https://www.ihcshotelconsulting.com/es/blog/tecnologia-e-innovacion-hotelera/ https://www.redalyc.org/pdf/433/43323186003.pdf https://improveverywhere.com/page/2/ https://www.tripadvisor.com.mx/Hotel_Review-g150793d152980-Reviews-Marriott_Puerto_Vallarta_Resort_Spa-Puerto_Vallarta.html 